\title{
Kooperative Unterrichtsettings: Technologische Tools zur Unterstützung von Lehrkräften
}

\author{
Anouschka van Leeuwen · Nikol Rummel
}

(C) Die Herausgeber und Springer Fachmedien Wiesbaden GmbH, ein Teil von Springer Nature 2019

Die Fähigkeit von Schülerinnen und Schülern, in Gruppen zu arbeiten und Probleme kooperativ zu lösen, erfährt weltweit zunehmende Aufmerksamkeit (OECD 2017). Kooperatives Lernen (KL; English „Collaborative Learning“) beschreibt Situationen, in denen zwei oder mehr Lernende zusammenarbeiten, um ein gemeinsames Lernziel zu erreichen (Dillenbourg 1999). Wiederholt wurde kooperativen Lernmethoden eine lernförderliche Wirkung bescheinigt (z. B., Kyndt et al. 2014). Darüber hinaus eröffnet kooperatives Lernen die Möglichkeit, Fähigkeiten wie Kreativität weiterzuentwickeln (Kumpulainen et al. 2013). Neben der Betrachtung von kooperativem Lernen als Instrument zur Förderung bestimmter lernbezogener Fähigkeiten, gilt die Kooperationsfähigkeit selbst als Schlüsselkompentenz in unserer heutigen Gesellschaft (Asterhan und Schwarz 2016). Jüngste PISA Ergebnisse bestätigen einen positiven Zusammenhang zwischen der Kooperationsfähigkeit von Schülerinnen und Schülern (SuS) und ihren jeweiligen Leistungen in Kernbereichen wie z. B. Lesen und Mathematik (OECD 2017). Für Lehrerende ergeben sich somit mannigfaltige Gründe, kooperative Lernsettings in ihrem Unterricht einzusetzen. In der Tat findet sich kooperatives Lernen auch den Standard-Curricula vieler europäischer Ländern wieder (European Parliament 2015).

Den unterschiedlichen Formen kooperativer Lernarrangements gemein ist die wichtige Rolle der Lehrkraft bei der Umsetzung, insbesondere mit Blick auf das Sicherstellen lernförderlicher Interaktionen innerhalb der Kleingruppen (Gillies et al. 2008). $\mathrm{Zu}$ den lernförderlichen Interaktionen zählen, in Abhängigkeit von der kon-

\footnotetext{
A. van Leeuwen $(\triangle)$

Department of Education, Utrecht University, Heidelberglaan 1, 3584CS Utrecht, Niederlande

E-Mail: A.vanLeeuwen@uu.nl

N. Rummel

Pädagogische Psychologie, Institut für Erziehungswissenschaft, Ruhr-Universität Bochum, Universitätsstraße 150, 44801 Bochum, Deutschland
} 
kreten kooperativen Lernmethode, beispielsweise das Stellen von Fragen, das Geben elaborierter Erklärungen und der Austausch von Argumenten (Asterhan und Schwarz 2016). Die Lehrkraft ist einerseits dafür verantwortlich, einen Rahmen zu schaffen, innerhalb dessen effektive Zusammenarbeit überhaupt erst entstehen kann, und anderseits dafür, die Interaktionen zwischen den SuS zu überwachen und anzuleiten (Gillies et al. 2008; Kaendler et al. 2015); zum Beispiel, indem sie interaktionsunterstützende Hinweise gibt, Fragen stellt und SuS dazu anregt, sich nicht nur auf inhaltlicher Ebene auszutauschen, sondern auch ihr gemeinsames Vorgehen bei der Problembearbeitung zum Gegenstand ihrer Diskussion zu machen (Van Leeuwen und Janssen 2019; Webb 2009). All diese Beispiele einer sinnvollen Anleitung durch Lehrkräfte erfordern ein enges Monitoring der Gruppenaktivitäten (Kaendler et al. 2015). Ohne adäquate Diagnose aktueller Kooperationsaktivitäten ist es der Lehrkraft kaum möglich, Unterstützungsbedarf zu erkennen bzw. einzuschätzen, welche Form der Unterstützung angemessen wäre. Wird der Unterstützungsbedarf einer Gruppe nicht wahrgenommen oder wird eine der Gruppe oder der Situation inadäquate Unterstützung gewählt (indem beispielsweise der Kooperationsprozess zu stark kontrolliert wird), so kann sich dies negativ auf die Zusammenarbeit auswirken (Van Leeuwen und Janssen 2019). Das Monitoring der Aktivitäten und Bedürfnisse von Lerngruppen ist somit eine entscheidende Aufgabe, mit der sich Lehrkräfte kontinuierlich befassen müssen.

Das Monitoring der Kooperation von SuS ist jedoch keineswegs trivial und stellt multiple Anforderungen an die Lehrkraft (Kaendler et al. 2015; Van Leeuwen et al. 2015). Die meisten kooperative Lernarrangements im Unterricht haben gemein, dass mehrere Gruppen zeitgleich angeleitet werden müssen und diese sowohl auf inhaltlicher als auch auf interaktionsbezogener Ebene der Unterstützung bedürfen. Zudem muss sich die Lehrkraft stets der Gefahr bewusst sein, die Zusammenarbeit womöglich zu einem ungünstigen Zeitpunkt zu unterbrechen und ist entsprechend gefordert, einen geeigneten Moment zur Intervention abzupassen. Es erscheint daher wenig überraschend, dass immer mehr Fortbildungsprogramme für Lehrende auf die Entwicklung erforderlicher Fähigkeiten zur Moderation von kooperativem Lernen abzielen (Kaendler et al. 2016).

In den vergangenen Jahren haben Technologien zur Unterstützung von kooperativen Lernarrangements im Schulunterricht an Bedeutung gewonnen (Stahl et al. 2006). Digitale Kooperationsszenarien bieten neue Möglichkeiten, SuS automatisiert in Echtzeit zu unterstützen und Lehrkräfte über die in Abhängigkeit vom kooperativen Lernarrangement relevanten Aspekte ihrer Zusammenarbeit von informieren. In digitalen Lernumgebungen werden in der Regel allen Gruppenaktivitäten aufgezeichnet, sodass eine Fülle von Daten analysiert und der Lehrkraft zur Verfügung gestellt werden kann, um sie im Monitoring des Kooperationsgeschehens zu unterstützen (Van Leeuwen 2015). In diesem Thementeil stehen solche Tools im Fokus des Interesses, die Lehrkräften Daten über die Kooperation der SuS in Form von Analysen oder Visualisierungen rückmelden. Derartige teacher orchestration tools versprechen eine effektivere Begleitung kooperativer Lernprozesse durch die Lehrkraft, da erwartet wird, dass die bereitgestellten Informationen dieser bei der Organisation und bei der Unterstützung der SuS während der Kooperation helfen (Prieto et al. 2011). 
Obgleich das Forschungsfeld noch jung ist, ist die Literatur zu teacher orchestration tools mittlerweile umfangreich genug, um relevante aktuelle Themen identifizieren zu können (Van Leeuwen und Rummel 2017), und um einen Thementeil wie diesen zu gestalten. Eines der aktuellen Forschungshemen betrifft die Funktionen, die ein orchestration tool beim Monitoring von Kooperationen durch eine Lehrkraft erfüllen kann. Während einige Tools lediglich Informationen bereitstellen, machen andere die Lehrkraft gezielt auf relevante Ereignisse aufmerksam; wiederrum andere geben sogar Empfehlungen für die Interpretation von und geeignete Unterstützungsmaßnahmen bei bestimmten Ereignissen. Diese verschiedenen Funktionen spiegeln die Phasen des Monitorings durch eine Lehrkraft wider: Lehrende müssen Ereignisse wahrnehmen, interpretieren und darauf basierend eine Entscheidung für adäquate Interventionen treffen (Kaendler et al. 2016; Van Es und Sherin 2002). Da Lehrkräfte sich in ihren Monitoring-Fähigkeiten in den verschiedenen Phasen unterscheiden (Van Es und Sherin 2002), erscheint es sinnvoll, wenn Fortbildungsprogramme ebenso wie orchestration tools auf die individuellen Fähigkeiten von Lehrenden zugeschnitten sind und genau an den Stellen ansetzen, an der die Lehrkraft beim Monitoring einer Kooperation abhängig vom Lernarrangement jeweils den größten Bedarf an Unterstützung aufweist. Diese Ideen sind vielversprechend, allerdings werfen sie auch eine Vielzahl an Fragen auf, beispielsweise wie eine Einschätzung der Monitoring-Fähigkeiten einer Lehrkraft erfolgen kann, um auf dieser Basis eine Entscheidung für ein möglichst optimal unterstützendes orchestration tool treffen zu können. Fragen wie dieser widmet sich u. a. dieser Thementeil.

\section{Aufbau des Thementeils}

Der Thementeil umfasst drei Beiträge, die Fragen des Einsatzes von teacher orchestration tools in kooperativen Unterrichtssettings in den Fokus nehmen. Der erste Beitrag, von Van Leeuwen und Rummel, gibt einen kurzen Überblick über den Forschungstand in diesem Feld. Die Autorinnen nehmen insbesondere solche Beiträge in den Blick, die eine Aussage darüber treffen, wie Lehrkräfte ihnen zur Verfügung gestellte orchestration tools nutzen und/oder wie sich der Einsatz dieser Tools auf die Anleitung der kooperierenden SuS auswirkt. Für die berücksichtigten Studien analysierten die Autorinnen unter anderem, welche Funktion die jeweils eingesetzten Tools übernahmen und wie die Studie konkret aussah. Aus den in den Übersichtsartikel aufgenommenen Beiträgen, die zum Großteil internationale Forschungsarbeiten darstellen, wird ersichtlich, dass in Deutschland in diesem Feld bislang nur wenig Forschung betrieben wurde. Dies ist vorrangig der Grund dafür, dass der Thementeil in englischer Sprache herausgegeben wird. Ein weiteres Ergebnis des Übersichtsartikels ist, dass die Mehrzahl der Studien qualitativer oder deskriptiver Natur ist, und nur wenige der Studien ein (quasi-)experimentelles Design realisieren. Dieser Befund steht im Einklang mit anderen Übersichtsarbeiten, die aus einer breiteren Perspektive auf die Rolle der Lehrkraft während der Zusammenarbeit von SuS blicken (Van Leeuwen und Janssen 2019; Kaendler et al. 2015; Webb 2009). Eine der Empfehlungen der Autorinnen für zukünftige Forschung ist daher, in systematische, experimentelle, groß angelegte Studien zu investieren, die das Forschungsfeld wei- 
ter erschließen und die Wirkung des Einsatzes von orchestration tools auf Lehrund Lern-Prozesse in kooperativen Unterrichtssettings untersuchen. Außerdem wird in zahlreichen Studien der Erläuterung der Entwicklung oder der technischen Details der orchestration tools viel Platz eingeräumt. Sie unterscheiden sich damit von klassischen empirischen Studien, wie sie in der Regel in Zeitschriften wie der Unterrichtswissenschaft veröffentlicht werden.

Der zweite Beitrag, von Swidan et al., ist ein Beispiel für diese Art von Studien, in dem Sinne, dass die Autoren die Details des orchestration tools sehr ausführlich beschreiben. In ihrer Studie untersuchten die Autoren die Wirkung eines orchestration tools mit Warnfunktion auf die Einblicke der Lehrkräfte in die kooperativen Lernprozesse. Es wird betrachtet, inwiefern Warnungen, die kritische Momente während der Zusammenarbeit von SuS anzeigen, Lehrkräften dabei helfen können, ein umfassenderes Bild vom Fortschritt parallel arbeitender Gruppen zu erhalten. Dabei zeigt sich allerdings auch, dass derartige Warnsignale in manchen Fällen die Wahrnehmung der Lehrkräfte beeinträchtigen. Die Autoren vermuten, dass dies insbesondere auf erfahrene Lehrkräfte zutrifft, die selbst bereits gut einschätzen können, wann und wie sie kooperierende SuS optimal unterstützen. Diese Lehrenden können die Warnsignale als störend empfinden. Die Ergebnisse der Studie bekräftigen die Idee, in adaptive orchestration tools zu investieren, die in Abhängigkeit von den individuellen Kompetenzen der Lehrkraft unterschiedliche Funktionen übernehmen.

Mit dem dritten und letzten Artikel des Thementeils, von Wiedmann et al., wird eine methodische Arbeit präsentiert, die zu der Frage beiträgt, auf welcher Basis der Zuschnitt eines orchestration tools auf den Unterstützungsbedarf einer Lehrkraft erfolgen kann. Die Autoren beschreiben und evaluieren ein Instrument zur Erfassung der Monitoring-Fähigkeiten von Lehrenden im Rahmen kooperativer Lernsettings. Das Instrument fordert die Lehrkräfte dazu auf, das Kooperationsgeschehen zu beschreiben und schaltet sich bei der Identifikation kritischer Momente ein, die bereits in dem Beitrag von Swidan et al. als neuralgische Punkte in Kooperationen beschrieben werden. Die Ergebnisse dieser Studie deuten auf das vielversprechende Potenzial des Instruments zur Einschätzung der Monitoring-Fähigkeiten von Lehrkräften hin.

Die drei Artikel beleuchten somit ein breites Spektrum an Fragen rund um den Einsatz und die Funktionsweisen von orchestration tools für Lehrende im Kontext von kooperativem Lernen im Unterricht und skizzieren den aktuellen Stand der Forschung in diesem Bereich, beispielsweise zur Frage, wie eingeschätzt werden kann, welche Art von Tool eine Lehrkraft am besten unterstützt. Wir hoffen, dass der Thementeil weitere Überlegungen und Forschungsarbeiten zu diesem Themenkomplex anregt.

\section{Literatur}

Asterhan, C.S.C., \& Schwarz, B.B. (2016). Argumentation for learning: Well-trodden paths and unexplored territories. Educational Psychologist, 51(2), 164-187. https://doi.org/10.1080/00461520.2016. 1155458.

Dillenbourg, P. (1999). What do you mean by collaborative learning? In P. Dillenbourg (Hrsg.), Collaborative-learning: Cognitive and computational approaches (S. 1-19). Oxford: Elsevier.

European Parliament (2015). Innovative schools: Teaching \& learning in the digital era. http://www. europarl.europa.eu/RegData/etudes/STUD/2015/563389/IPOL_STU(2015)563389_EN.pdf 
Gillies, R. M., Ashman, A., \& Terwel, J. (Hrsg.). (2008). The teacher's role in implementing cooperative learning in the KLassroom. New York: Springer.

Kaendler, C., Wiedmann, M., Rummel, N., \& Spada, H. (2015). Teacher competencies for the implementation of collaborative learning in the KLassroom: A framework and research review. Educational Psychology Review, 27(3), 505-536.

Kaendler, C., Wiedmann, M., Rummel, N., Leuders, T., \& Spada, H. (2016). Monitoring student interaction during collaborative learning: Design and evaluation of a training program for pre-service teachers. Psychology Learning \& Teaching, 15(1), 44-64.

Kumpulainen, K., Mikkola, A., \& Jaatinen, A.-M. (2013). The chronotopes of technology-mediated creative learning practices in an elementary school community. Learning, Media and Technology, 39, 53-74. https://doi.org/10.1080/17439884.2012.752383.

Kyndt, E., Raes, E., Lismont, B., Timmers, F., Dochy, F., \& Cascallar, E. (2014). A meta-analysis of the effects of face-to-face cooperative learning. Do recent studies falsify or verify earlier findings? Educational Research Review, 10, 133-149. https://doi.org/10.1016/j.edurev.2013.02.002.

OECD (2017). Collaborative problem solving. https://www.oecd.org/berlin/publikationen/PISA_ ProblemSolving_Ergebnisse.pdf

Prieto, L.P., Holenko Dlab, M., Gutierrez, I., Rrez, N. A., Abdulwahed, M., \& Balid, W. (2011). Orchestrating technology enhanced learning: A literature review and a conceptual framework. International Journal of Technology Enhanced Learning, 3(6), 583. https://doi.org/10.1504/IJTEL.2011.045449.

Stahl, G., Koschmann, T., \& Suthers, D. (2006). Computer-supported collaborative learning: An historical perspective. In R. K. Sawyer (Hrsg.), Cambridge handbook of the learning sciences (S. 409-426). Cambridge: Cambridge University Press.

Van Es, E.A., \& Sherin, M.G. (2002). Learning to notice: Scaffolding new teachers' interpretations of KLassroom interactions. Journal of Technology and Teacher Education, 10(4), 571-596.

Van Leeuwen, A. (2015). Learning Analytics to support teachers during synchronous CSKL: Balancing between overview and overload. Journal of Learning Analytics, 2, 138-162. https://doi.org/10.18608/ jla.2015.22.11.

Van Leeuwen, A., \& Janssen, J. (2019). A systematic review of teacher guidance during collaborative learning in primary and secondary education. Educational Research Review, 27, 71-89. https://doi. org/10.1016/j.edurev.2019.02.001.

Van Leeuwen, A., \& Rummel, N. (2017). Teacher regulation of collaborative learning: Research directions for learning Analytics dashboards. In B. K. Smith, M. Borge, E. Mercier \& K. Y. Lim (Hrsg.), Proceedings of the 12th international conference on computer-supported collaborative learning (Bd. 2, S. 805-806). Philadelphia: International Society of the Learning Sciences.

Van Leeuwen, A., Janssen, J., Erkens, G., \& Brekelmans, M. (2015). Teacher regulation of multiple computer-supported collaborating groups. Computers in Human Behavior, 52, 233-242. https://doi.org/ 10.1016/j.chb.2015.05.058.

Webb, N.M. (2009). The teacher's role in promoting collaborative dialogue in the KLassroom. British Journal of Educational Psychology, 79, 1-28. https://doi.org/10.1348/000709908X380772. 